\title{
Workplace asthma
}

\section{L'asthme en milieu de travail}

I $\mathrm{n}$ the present issue of the Canadian Respiratory Journal, Demir et al (1) explore the topic of work-related asthma in a very interesting way. Their data were derived from a previous large multicentre study of asthma prevalence in Canada (2), which has been previously exploited to obtain a wide variety of interesting results (3), including an estimate of occupational asthma (4). Demir et al (1) analyzed results from the Montreal (Quebec) centre in greater detail than had been done previously.

The study recruited subjects by random community sampling, which is the best way to approach the issue of work-related asthma, because it is not subject to the 'healthy worker effect' that bedevils workplacebased studies of asthma or any other disease. This effect notes the probability that people who have trouble in the workplace are likely to change their work and not be available for study. The community-based sample was studied in a two-step format. In Montreal, approximately 2900 people filled out a questionnaire related to asthma, over $85 \%$ of those who were asked to do so. Of these, 498 people visited the laboratory and underwent further study, including allergy skin tests, measures of bronchial reactivity and a detailed occupational questionnaire. These 498 subjects represented only $36 \%$ of those asked to attend the laboratory, and were more likely to have asthma symptoms and less likely to smoke than the larger sample. However, the exposure of the smaller group to putative occupational hazards was similar to that of the larger group, so that the differences between groups should not have affected the relationship between exposure and asthma. The occupational questionnaire assessed exposure over the lifetime of the subjects using a checklist involving 22 industries, 19 individual job titles and 22 specific agents, with workplace exposure of at least three months being required for a positive answer.

Positive responses for asthma were regarded as current wheeze $(23.7 \%$ of respondents), history of asthma attacks or treatment $(13 \%)$, or airways hyperreactivity $(15 \%)$; the last was rather stringent, being a response to $2 \mathrm{mg} / \mathrm{mL}$ of methacholine or less. Exposures were divided into two general categories: irritants and sensitizers. The former were relatively low molecular weight compounds, including dyes, hardeners and metals, and the latter were of higher molecular weight, including vegetable dusts, fur and enzymes. Exposures to sensitizers were reported by approximately $57 \%$ of the intensively studied group, while exposures to irritants occurred in approximately $10 \%$. Asthma responses were related to exposures.

Several striking results are reported. First, all exposures that related to asthma had occurred in the past and not in the subject's current work (the healthy worker effect). Second, exposure to irritants was related to current wheeze, while exposure to sensitizers was related to airways hyperreactivity. Indeed, subjects with asthma diagnoses or airway reactivity had a negative ans le présent numéro de la Revue canadienne de pneumologie, Demir et coll. (1) explorent le thème de l'asthme en milieu de travail d'une façon très intéressante. Leurs données proviennent d'une grande étude multicentrique sur la prévalence de l'asthme au Canada (2), dont une analyse antérieure avait produit une vaste gamme de résultats qui ont retenu l'attention (3), entre autres, une estimation de l'asthme professionnel (4). Demir et coll. (1) ont voulu analyser encore plus en détail les résultats du centre de Montréal (Québec).

L'étude a recruté ses sujets par échantillonnage aléatoire dans la communauté, ce qui est la meilleure façon d'aborder la question de l'asthme professionnel, puisqu'il échappe ainsi au phénomène du « travailleur en bonne santé » qui nuit aux études en milieu de travail sur l'asthme ou sur toute autre maladie. En effet, ce phénomène fait ressortir la probabilité que les gens qui ont des problèmes en milieu de travail risquent fort de changer de boulot et de ne pas être disponibles pour participer aux études. L'échantillon basé dans la communauté a été étudié en deux étapes. À Montréal, environ 2900 personnes ont répondu à un questionnaire sur l'asthme, soit plus de $85 \%$ des personnes qui avaient été invitées à le faire. Parmi ces gens, 498 personnes se sont présentées au laboratoire et ont subi des examens plus approfondis, y compris des tests d'allergie cutanés, des mesures de leur réactivité bronchique et un questionnaire professionnel détaillé. Ces 498 sujets ne représentaient que 36 \% de ceux qui avaient été invités à se rendre au laboratoire et étaient plus susceptibles de souffrir de symptômes d'asthme et moins susceptibles de fumer que l'échantillon général. Or, l'exposition du groupe restreint à des risques professionnels potentiels s'est révélée similaire à celle de l'échantillon général, de sorte que les différences entre les groupes n'ont pas modifié le lien entre l'exposition et l'asthme. Le questionnaire professionnel visait à évaluer le degré d'exposition à vie des sujets au moyen d'une liste comportant 22 industries, 19 professions et 22 agents spécifiques; l'exposition en milieu de travail devait avoir duré au moins trois mois pour que l'on puisse qualifier la réponse de positive.

Les réponses jugées positives à l'égard de l'asthme ont été les suivantes : sibilances à l'examen (23,7 \% des répondants), antécédents de crises d'asthme ou de traitements anti-asthmatiques (13\%) ou hyperréactivité bronchique (15\%). Ce dernier critère était plutôt strict, correspondant à une réaction à $2 \mathrm{mg} /$ mL de méthacholine ou moins. Les types d'exposition ont été divisés en deux catégories générales : irritants et sensibilisateurs. Le premier groupe faisait référence à des substances de poids moléculaires relativement faibles, soit teintures, durcisseurs et métaux, et le second, à des produits de poids moléculaires plus élevés, notamment, poussières végétales, fourrure et enzymes. L'exposition à des sensibilisateurs a été signalée par environ $57 \%$ du groupe étudié de manière plus intensive, 
association with exposure to irritants, indicating that these people avoided such exposure, another illustration of the healthy worker effect. All three of the asthma outcomes assessed related to work in the same way whether the subjects had had asthma as a child or not; thus, a history of childhood asthma did not influence the work relatedness of the asthma. This means that a history of childhood asthma should not influence judgments as to whether a given individual does or does not have work-related asthma. People who have had asthma as children are more likely to get it as adults, but this does not indicate that the adult disease is not work-related. Finally, Demir et al (1) conclude that work-related asthma could account for up to one-third of adult-onset asthma, similar to the figure derived from a previous analysis of the entire dataset (4). This is indeed food for thought.

Finally, I cannot close without paying tribute to Canadian investigators in the field of occupational asthma. A colleague and collaborator of mine, Jure Manfreda, was the principle investigator of the original asthma prevalence study. The senior author of the Demir paper is Margaret Becklake, a towering figure in respiratory epidemiology who is an international expert in this field. One could also argue that the founding 'fathers' of studies of occupational asthma are Moira Chan-Yeung of Vancouver, British Columbia, and Jean-Luc Malo of Montreal (5). It should be noted that Dr Malo publishes another paper in occupational asthma in the present issue of the Canadian Respiratory Journal (6).

Nick R Anthonisen MD Editor-in-Chief, Canadian Respiratory Journal

\section{REFERENCES}

1. Demir A, Joseph L, Becklake MR. Work-related asthma in Montreal, Quebec: Population attributable risk in a communitybased study. Can Respir J 2008;15:406-12.

2. Manfreda J, Becklake MR, Sears MR, et al. Prevalence of asthma symptoms among adults aged 20-44 years in Canada. CMAJ 2001;164:995-1001.

3. Al-Hazmi M, Wooldrage K, Anthonisen NR, et al. Airflow obstruction in young adults in Canada. Can Respir J 2007;14:221-7.

4. Johnson AR, Dimich-Ward HD, Manfreda J, et al. Occupational asthma in adults in six Canadian communities. Am J Respir Crit Care Med 2000;162:2058-62.

5. Chan-Yeung M, Malo JL. Aetiological agents in occupational asthma. Eur Respir J 1994;7:346-71.

6. Malo J-L, L'Archeveque J, Ghezzo H. Direct costs of occupational asthma in Quebec between 1988 and 2002. Can Respir J 2008;15:413-6. tandis que l'exposition aux irritants a affecté environ $10 \%$ des sujets. Les réponses de type asthmatique étaient en lien avec les expositions.

Plusieurs résultats frappants ont été rapportés. Tout d'abord, toutes les expositions liées à l'asthme étaient choses du passé et n'affectaient pas le travail actuel des sujets (phénomène du travailleur en bonne santé). Ensuite, l'exposition aux irritants était liée aux sibilances, tandis que l'exposition aux sensibilisateurs était liée à l'hyperréactivité bronchique. En effet, les sujets qui avaient un diagnostic d'asthme ou d'hyperréactivité bronchique présentaient un lien négatif avec l'exposition aux irritants, ce qui indique que ces gens évitaient ce type d'exposition, autre illustration du phénomène du travailleur en bonne santé. Les trois paramètres liés à l'asthme évalués avaient un lien similaire avec le travail, que les sujets aient souffert d'asthme infantile ou non. Ainsi, les antécédents d'asthme infantile n'ont pas influé sur le lien de l'asthme avec le travail. Cela signifie que des antécédents d'asthme infantile ne devraient pas influer sur le fait qu'une personne donnée ait ou non un asthme professionnel. Les gens qui ont souffert d'asthme durant l'enfance sont plus susceptibles d'en souffrir à l'âge adulte, mais cela n'est pas un signe que la maladie soit liée au travail chez l'adulte. En terminant, Demir et coll. (1) affirment que l'asthme professionnel pourrait représenter jusqu'à un tiers des cas d'asthme qui se déclenchent à l'âge adulte, ce qui coïncide avec les résultats d'une analyse précédente sur l'ensemble des données (4). Il y a donc effectivement matière à réflexion.

Je ne saurais conclure sans rendre hommage aux chercheurs canadiens qui travaillent dans le domaine de l'asthme professionnel. Mon collègue et collaborateur Jure Manfreda était l'investigateur principal de l'étude originale sur la prévalence de l'asthme. L'auteur principal de l'article de Demir est Margaret Becklake, une sommité internationale en épidémiologie des maladies respiratoires. On pourrait également affirmer que les premiers instigateurs des études sur l'asthme professionnel sont Moira Chan-Yeung, de Vancouver, en Colombie-Britannique, et Jean-Luc Malo, de Montréal (5). Fait à noter, le Dr Malo publie un autre article sur l'asthme professionnel dans le présent numéro de la Revue canadienne de pneumologie (6).

Nick R Anthonisen MD Rédacteur en chef, Revue canadienne de pneumologie

\section{RÉFÉRENCES}

1. Demir A, Joseph L, Becklake MR. Work-related asthma in Montreal, Quebec: Population attributable risk in a communitybased study. Can Respir J 2008;15:406-12.

2. Manfreda J, Becklake MR, Sears MR et coll. Prevalence of asthma symptoms among adults aged $20-44$ years in Canada. CMAJ 2001;164:995-1001.

3. Al-Hazmi M, Wooldrage K, Anthonisen NR et coll. Airflow obstruction in young adults in Canada. Can Respir J 2007;14:221-7.

4. Johnson AR, Dimich-Ward HD, Manfreda J et coll. Occupational asthma in adults in six Canadian communities. Am J Respir Crit Care Med 2000;162:2058-62.

5. Chan-Yeung M, Malo JL. Aetiological agents in occupational asthma. Eur Respir J 1994;7:346-71.

6. Malo J-L, L'Archeveque J, Ghezzo H. Direct costs of occupational asthma in Quebec between 1988 and 2002. Can Respir J 2008;15:413-6. 


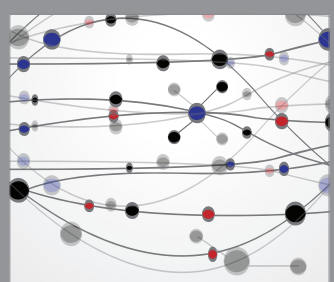

The Scientific World Journal
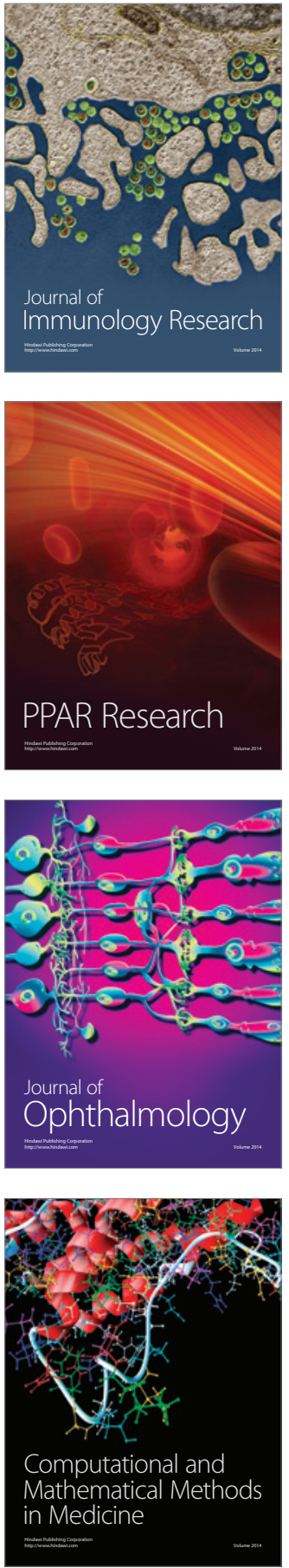

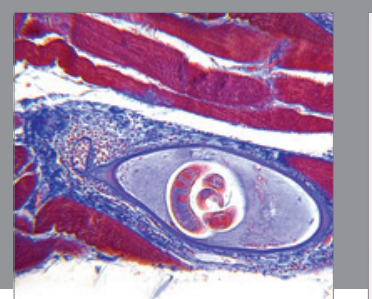

Gastroenterology Research and Practice

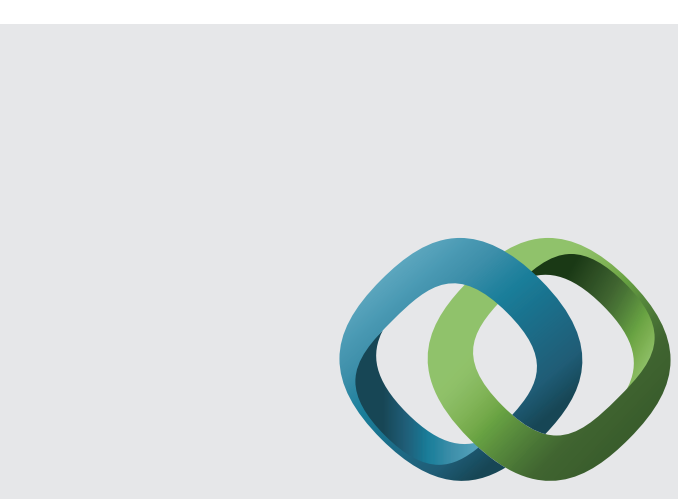

\section{Hindawi}

Submit your manuscripts at

http://www.hindawi.com
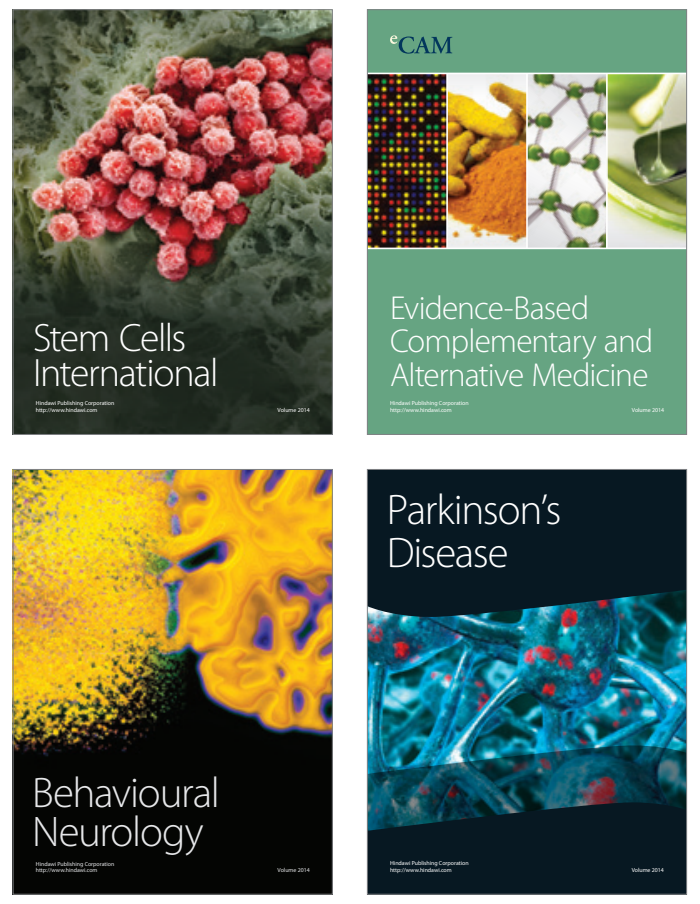
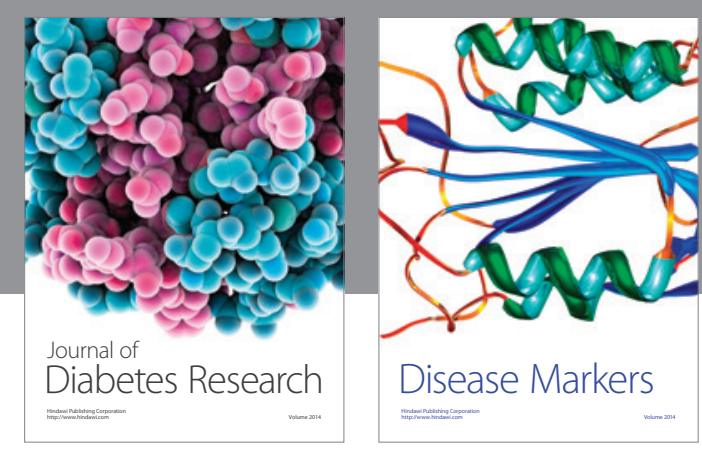

Disease Markers
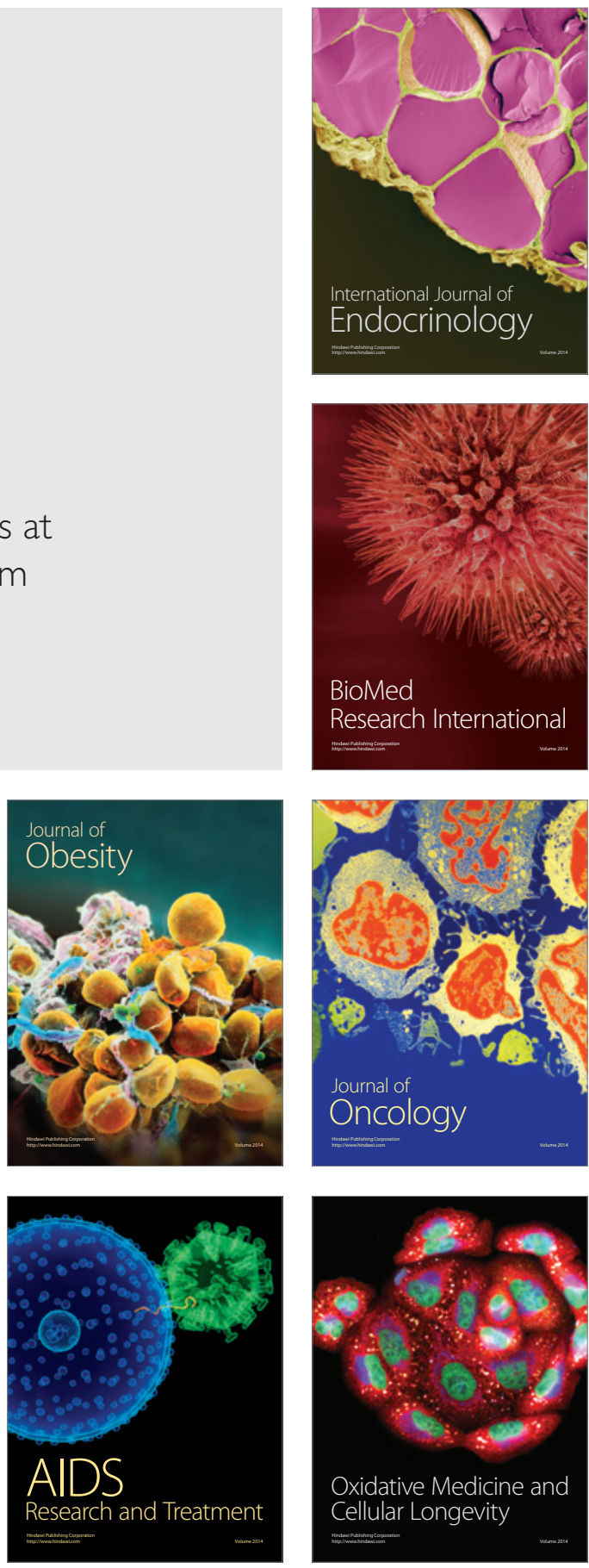